\title{
Preoperative fibrinogen to prealbumin ratio as a novel predictor for clinical outcome of hepatocellular carcinoma
}

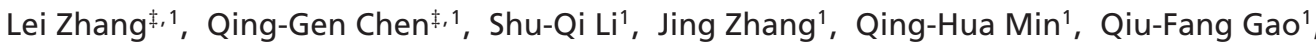 \\ Fan Sun ${ }^{1}$, Yu-Huan Jiang ${ }^{1}$, Xiao-Zhong Wang ${ }^{1}$ \& Hou-Qun Ying ${ }^{*, 1}$ \\ ${ }^{1}$ Department of Clinical Laboratory, Jiangxi province Key Laboratory of Laboratory Medicine, The Second Affiliated Hospital of \\ Nanchang University, Nanchang 330006, Jiangxi, PR China \\ *Author for correspondence: Tel.: +86 0791 86300410; Fax: +86 0791 86300410; yinghouqun2013@163.com \\ ${ }^{\ddagger}$ Authors contributed equally
}

\begin{abstract}
Aim: To investigate prognostic value of preoperative inflammatory biomarkers in hepatocellular carcinoma (HCC). Patients \& methods: Preoperative circulating fibrinogen, prealbumin, fibrinogen to prealbumin ratio (FPR), neutrophil to lymphocyte ratio, derived neutrophil to lymphocyte ratio, lymphocyte to monocyte ratio, platelet to lymphocyte ratio were detected and calculated in $230 \mathrm{HCC}$ patients. X-tile software, Kaplan-Meier curve, Cox regression, time-dependent receiver-operating characteristic were used to explored prognostic roles of them in HCC. Results: Multivariate Cox regression showed that high FPR was significantly associated with decreased recurrence-free survival $(p=0.034)$ and overall survival $(p<0.001)$ within HCC patients. FPR generated the largest area under curve of time-dependent receiver-operating characteristic comparing to the other biomarkers. Overall survival of HCC patients receiving chemotherapy was superior to the cases without receiving chemotherapy only in high FPR subgroup $(p=0.028)$. Conclusion: Preoperative FPR was superior to other biomarkers to independently predict survival of HCC patients, and it could identify the patients who could benefit from adjuvant chemotherapy.
\end{abstract}

First draft submitted: 17 May 2018; Accepted for publication: 31 July 2018; Published online: 24 August 2018

Keywords: FPR • HCC • inflammation

Hepatocellular carcinoma (HCC) is the fourth most common malignancy and the third most common cause of cancer-related death in China [1]. Currently, surgical resection and the followed adjuvant chemotherapy remain the main strategies for treatment of these patients [2]. However, approximately $60-70 \%$ of the treated patients were occurred recurrence or distant metastasis in the following 5 years [3]. Therefore, it is important to explore economical and reliable biomarker to predict the survival of HCC patients and to stratify the patients who may benefit from adjuvant chemotherapy after hepatectomy.

Increasing evidences have suggested that chronic inflammation are closely related to occurrence, progression, metastasis of malignancies, including HCC [4-6]. Several inflammation-based prognostic scores, such as neutrophil to lymphocyte ratio (NLR), derived neutrophil to lymphocyte ratio (dNLR), lymphocyte to monocyte ratio (LMR), platelet to lymphocyte ratio (PLR), have been developed to predict postoperative recurrence and overall survival (OS) in HCC patient [7-9]. However, these indicators are not widely used in clinic for the limited predictive efficacy of them. Therefore, a more effective biomarker is warrant to predict the prognosis of HCC patients.

Recent studies have revealed that fibrinogen (Fib) and its derivative peptides play proinflammatory roles in solid cancer [10]. Moreover, emerging evidence show that elevated Fib is associated with poor OS and tumor-free survival [11-13]. In addition to Fib, prealbumin (pAlb) is a common nutritional indicator and low preoperative pAlb level is a negative independent prognostic factor for cancer-specific survival [14-16]. Hence, we hypothesized that circulating Fib to pAlb ratio (FPR) would be a prospective biomarker to predict the survival of HCC.

In this study, we investigated the prognostic roles of prospective circulating Fib, pAlb, FPR, NLR, dNLR, PLR and LMR in 230 HCC patients. We first revealed that circulating FPR as a novel biomarker to predict the 
recurrence-free survival (RFS) and OS of HCC patients with treatment of hepatectomy and to classify the patients who could benefit from adjuvant chemotherapy.

\section{Patients \& methods}

Two hundred and thirty eligible HCC patients were enrolled according to the inclusion and exclusion criteria in our study. All the enrolled patients received hepatectomy from January 2011 to December 2013 at the Second Affiliated Hospital of Nanchang University. All of them were clinical newly confirmed by contrast-enhanced computed tomography, MRI and pathologic biopsy according to the Asian Pacific Association for the Study of the Liver guideline [17]. On the contrary, those individuals were excluded in accordance with the following exclusion criteria: patient with cholangiocarcinoma or mixed HCC; patient with clinical evidence of infection, inflammationrelated disease, autoimmune disease or blood system disease in recent 3 months; patient with hepatic insufficiency; patient with previous history of malignancy; patient with previous treatment before surgery, such as ablation, transcatheter arterial chemoembolization, chemotherapy or radiotherapy; patient with incomplete follow-up data. The written informed consents were obtained from all patients and the study protocol was approved by the Ethical Committee of the hospital.

The baseline characteristics, gender, alcohol and cigarette intake, hypertension, diabetes, Barcelona Clinic Liver Cancer Group (BCLC) stage, maximal tumor diameter, histological differentiation, histological type and clinical therapy were gathered from the medical record of each individual. Peripheral blood sample was collected at 7:30 to $9: 30 \mathrm{am}$ in 1 week before surgical resection. Plasma and serum samples were obtained by centrifuging in 3000 revolution per minute, and peripheral cell counting, serum alpha-fetoprotein (AFP), pAlb and plasma Fib were detected within $3 \mathrm{~h}$ after the sample preparation. SYSMEX XE-2100 machine (Sysmex, Tokyo, Japan) with the lectric resistance method, SIEMENS ADVIA Centaur CP machine (Siemens, Erlangen, Germany) with the chemiluminescent immunoassay method, OLYMPUS AU5400 machine (Beckman Coulter, Tokyo, Japan) with the immunoturbidimetric assay method and SYSMEX CA-7000 machine (Sysmex) with the Clauss method were used to measure the counts of peripheral cell, serum AFP, pAlb and plasma Fib concentration, respectively. The inter- and intrabatch coefficients of variation of Fib and pAlb kits were within the controllable range. We calculated FPR using the following formula: FPR $=\mathrm{Fib}^{*} 1000 / \mathrm{pAlb}$.

All recruited patients were followed up every 3 months by means of retrieval medical record, telephone, email and correspondence until 31 December 2016. Three years' RFS and OS were the main end points in present study, and two of them were calculated from surgical operative time to recurrence and the dead or the last follow-up.

In this study, continuous variables were presented as median (entire range) or mean \pm standard deviation (SD). Pearson $\chi^{2}$ test, Fisher exact test and Kruskal-Wallis test were used to compare the difference in categorical data. Student's t-test, Mann-Whitney U test were selected to compare the difference in quantitative data. The optimal cut-off points of each candidate ratio were measured by X-tile software. Survival difference and prognostic role of the each ratio were estimated by Kaplan-Meier curve with log-rank test, univariate and multivariate Cox proportional regression. Time-dependent receiver-operating characteristic (ROC) curve was used to compare survival-predicted efficacy of each ratio. All the statistics were two-sided, and $\mathrm{p}<0.05$ was considered as statistical significance, and all of them were carried out using R 3.3.2 software (Institute for Statistics and Mathematics, Vienna, Austria), and SPSS 22.0 software (SPSS, IL, USA).

\section{Result}

According to inclusion and exclusion criteria, 230 eligible patients were included in our study, and their clinical characteristics were summarized in Table 1. One hundred and ninety-two (83.50\%), $32(13.90 \%)$ and six (2.60\%) patients were diagnosed as BCLC stage A, stage B and stage $\mathrm{C}$, respectively. The median tumor size was $5(0.60-$ $17.00) \mathrm{cm}$, and 128 patients $(55.70 \%)$ had tumor size less than $5 \mathrm{~cm}$. One hundred and eighty-eight $(81.70 \%)$ patients showed well differentiated and the number of patients with single tumor was 197 (85.70\%). HBV and elevated AFP $(>400 \mu \mathrm{g} / \mathrm{l})$ were detected in $192(83.50 \%)$ patients and $90(39.10 \%)$ patients, respectively. No patient was observed with HCV infection. The median or mean of Fib, pAlb, NLR, dNLR, PLR, LMR, FPR were 2.45 (1.08-6.06) mg/dl, $160.90 \pm 56.30 \mathrm{mg} / \mathrm{l}, 1.99$ (0.14-15.30), 2.4 (0.35-16.18), 96.47 (18.25-775.76), 4.06 (0.95-23.10), 16.51 (6.45-154.19), respectively.

The respective optimal cut-off points of Fib, pAlb and FPR were $3.3 \mathrm{mg} / 1,152.5 \mathrm{mg} / \mathrm{l}$ and 15.6 for OS prediction in HCC patients (Figure 1). In accordance with the cut-off point, the included patients were divided into high and low subgroups stratified by each biomarker. Results of Kaplan-Meier curve, univariate and multivariate Cox 
(A)

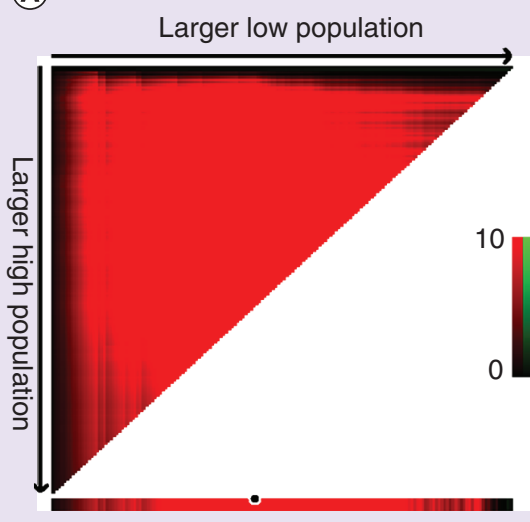

(D)

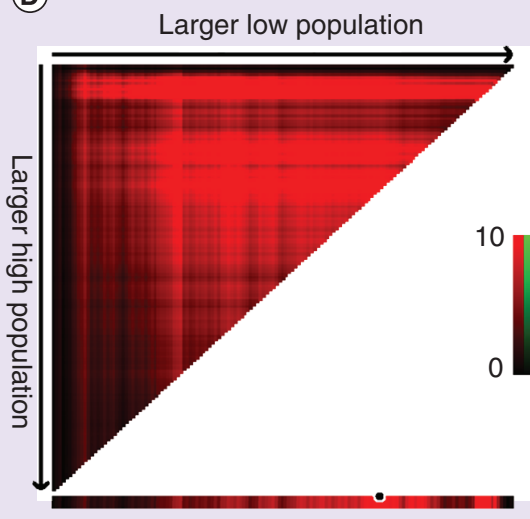

(G)

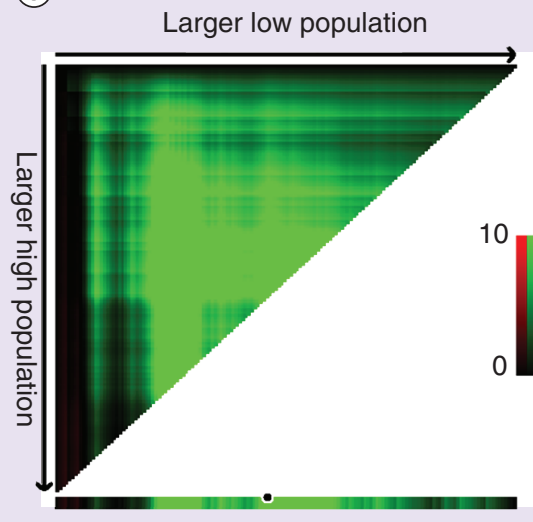

(B)

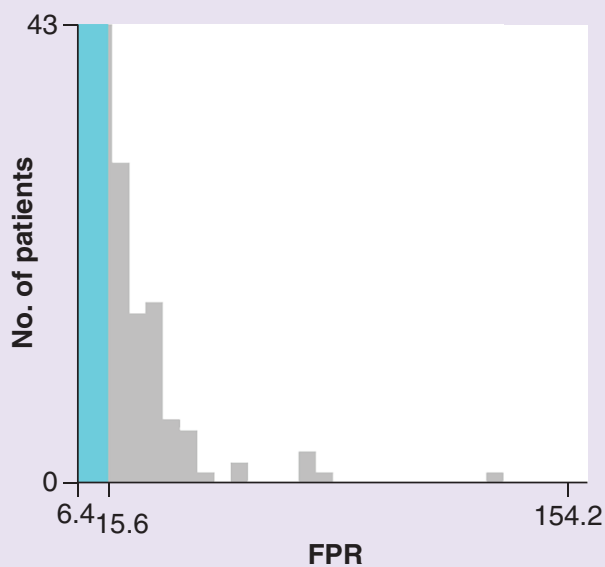

(E)

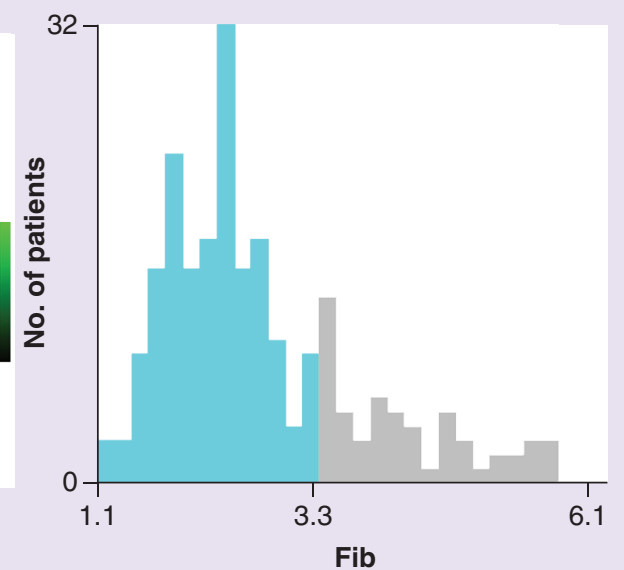

(H)

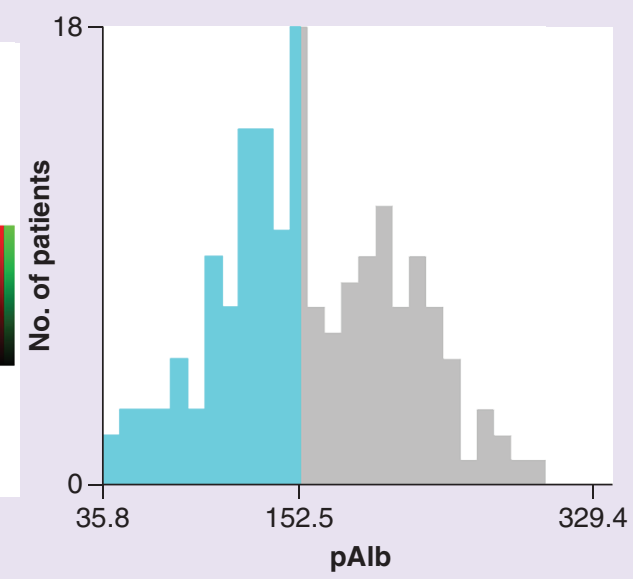

(C)

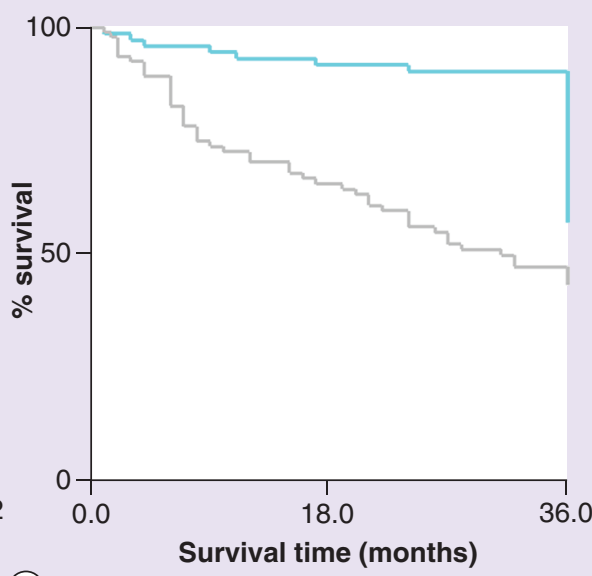

(F)

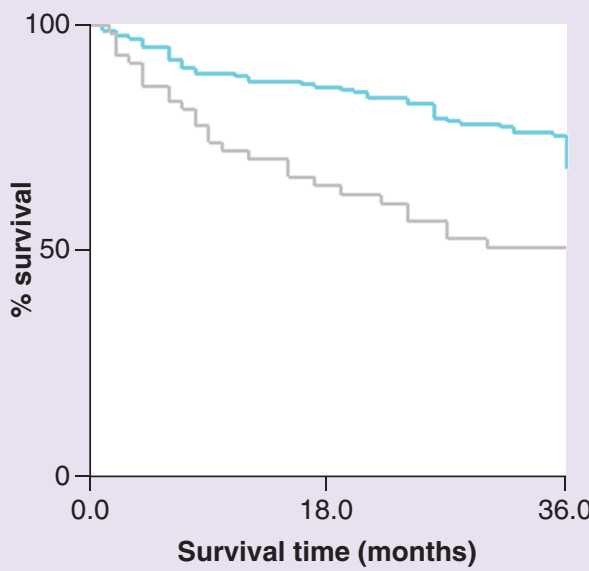

(I)

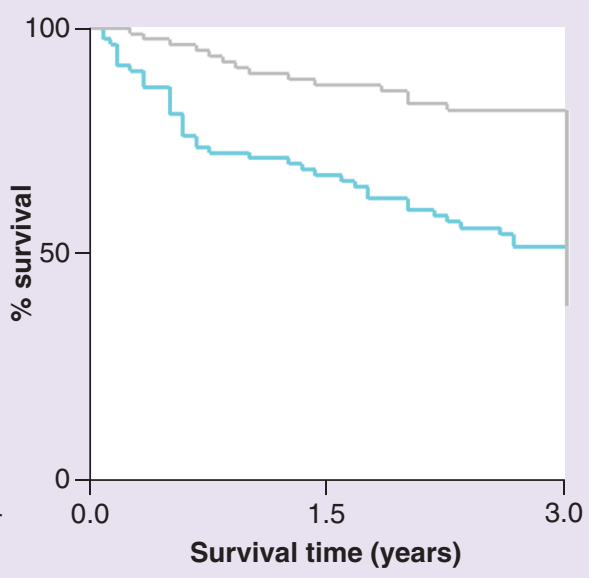

Figure 1. The optimal cut-off value for fibrinogen to prealbumin ratio determined by X-title software for 3 years' overall survival in hepatocellular carcinoma patients. (A, D \& G) The data were represented graphically in a right-triangular grid where each point represents the data from a given set of divisions. The plots showed the $\chi^{2}$ log-rank values produced, dividing them into two groups by the cut-off point. The optimal cutpoints, 15.6, 3.3 and 152.5, were determined by locating the brightest pixel on the X-tile plot. The distribution of number of patients was shown on the histogram $(B, E \& H)$ and corresponding populations were displayed on the Kaplan-Meier curve (C, F \& I). (A-C) The optimal cut-off value of FPR; (D-F) The optimal cut-off value of Fib; (G-I) The optimal cut-off value of pAlb.

Fib: Fibrinogen; FPR: Fibrinogen to prealbumin ratio; pAlb: Prealbumin. 
Table 1. The clinical characteristics of 230 hepatocellular carcinoma patients.

\begin{tabular}{|c|c|c|}
\hline Variables & Total patients $(\mathrm{n}=230)$ & No. of patients (\%) \\
\hline Gender (Male) & 193 & 83.90 \\
\hline Age (year) & & $51.60 \pm 12.20$ \\
\hline Alcohol (yes) & 56 & 24.30 \\
\hline Hypertension (yes) & 22 & 9.60 \\
\hline Diabetes (yes) & 16 & 7.00 \\
\hline Radiotherapy (yes) & 23 & 10.00 \\
\hline Differentiation (well) & 188 & 81.70 \\
\hline HCV infection (yes) & 0 & 0.00 \\
\hline HBV infection (yes) & 192 & 83.50 \\
\hline BCLC stage $(A)$ & 192 & 83.50 \\
\hline $\operatorname{AFP}(\leq 400 \mu \mathrm{g} / \mathrm{l})$ & 136 & 59.20 \\
\hline Fib $(\mathrm{mg} / \mathrm{dl})$ & & $2.45(1.08-6.06)$ \\
\hline pAlb (mg/l) & & $160.50 \pm 56.30$ \\
\hline NLR & & $1.99(0.14-15.30)$ \\
\hline dNLR & & $2.40(0.35-16.18)$ \\
\hline PLR & & $96.47(18.25-775.76)$ \\
\hline LMR & & $4.12(0.95-23.10)$ \\
\hline FPR & & $16.51(6.45-154.19)$ \\
\hline
\end{tabular}

regression of the inflammatory biomarkers within our study were described in Figure 2 and Table 2. The results showed that gender, age, cigarette and alcohol intake, hypertension and diabetes status as well as HBV infection were not associated with RFS or OS in HCC patients (all p > 0.05). According to adjuvant treatment, chemotherapy was significantly associated with OS (adjusted hazard ratio [HR]: 0.485; 95\% CI: 0.291-0.808) of the patient. Multiple tumor number (adjusted HR: 3.209; 95\% CI: 1.903-5.414) and low LMR (adjusted HR: 2.050; 95\% CI: 1.060-3.965) were only related to poor RFS in the patients, however, high BLCL stage (adjusted HR: 3.168; 95\% CI: $1.705-5.886$ ) patient and low pAlb (adjusted HR: 2.345; 95\% CI: 1.252-4.393) were significantly associated with short OS in the patients. RFS and OS of patients with poor cell differentiation (adjusted HR: 2.207; 95\% CI: 1.241-3.926 for RFS, adjusted HR: 2.327; 95\% CI: 1.274-4.253 for OS), large tumor size (adjusted HR: 1.955; 95\% CI: 1.243-3.075 for RFS, adjusted HR: 1.904; 95\% CI: 1.093-3.319 for OS) and high FPR (adjusted HR: 1.765; 95\% CI: 1.044-2.987 for RFS, adjusted HR: 4.155; 95\% CI: 2.059-8.387 for OS) were significantly poorer than the patients with well differentiation, small tumor size and low FPR, respectively.

To further compare the prognostic values of independent prognostic factors, the time-dependent ROC was carried out, and the results showed that area under curves (AUCs) of FPR reached up to 0.709 and 0.741 for predicting RFS and OS in 36th follow-up months, and all of them were higher than the AUCs of the other biomarkers in predicting recurrence and death within HCC patients (Figure 3). For this, we tried to investigate the possible association of FPR with clinical characteristics, and the results showed that no obvious difference of cigarette and alcohol intake, HBV infection and AFP was observed in high and low FPR subgroups (all p $>0.05$ ). Whereas high FPR was significantly associated with large tumor size $(\mathrm{p}<0.001)$ and BCLC B/C stage $(\mathrm{p}<0.05$; Figure 4). Furthermore, we observed that the survival of surgical HCC patients receiving chemotherapy was significant longer than the patients without the treatment in high FPR subgroup ( $\mathrm{p}=0.028)$. However, no survival difference was observed between the patients with or without chemotherapy in low FPR subgroup ( $\mathrm{p}=0.116$; Figure 5). 


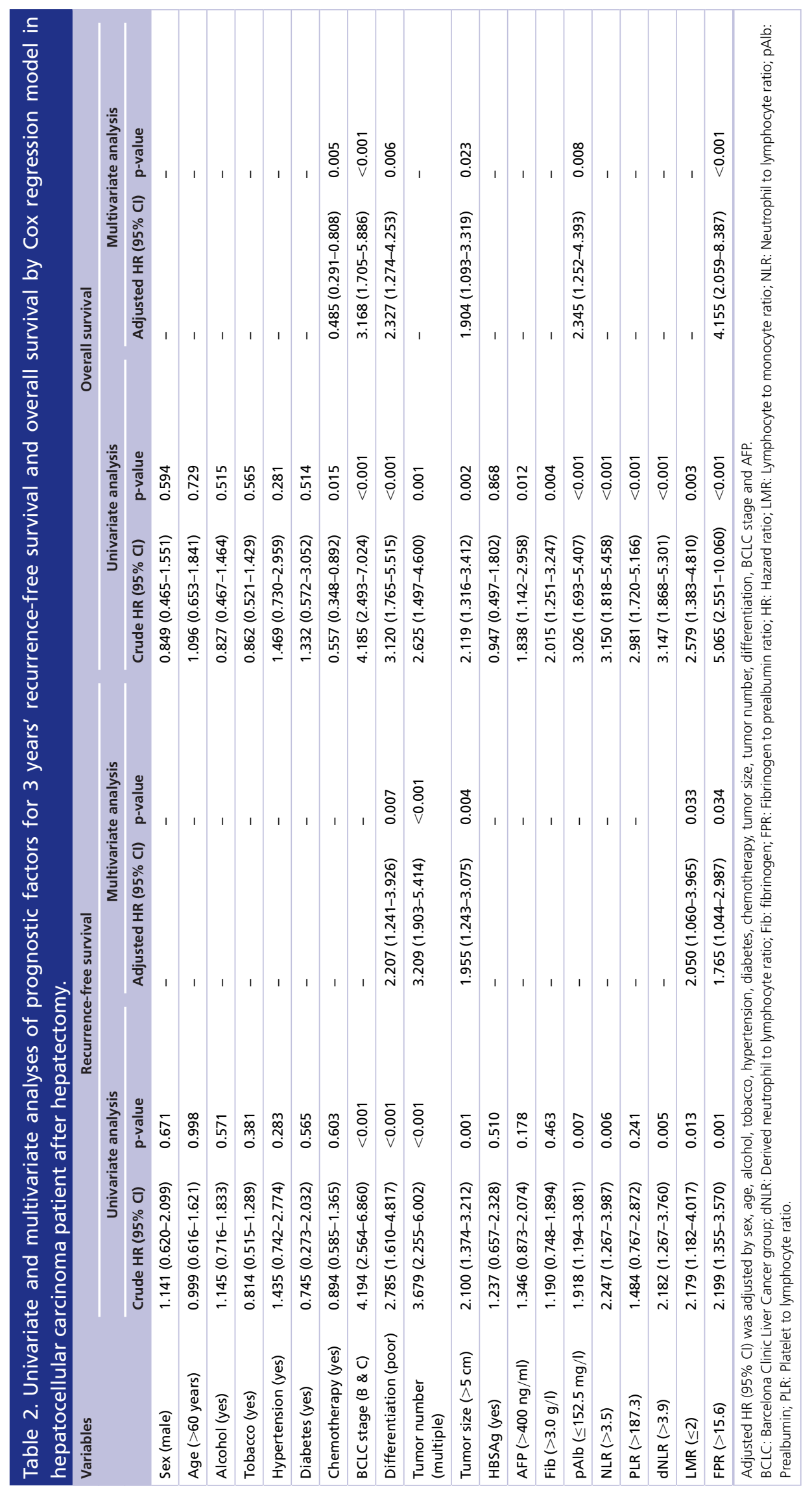




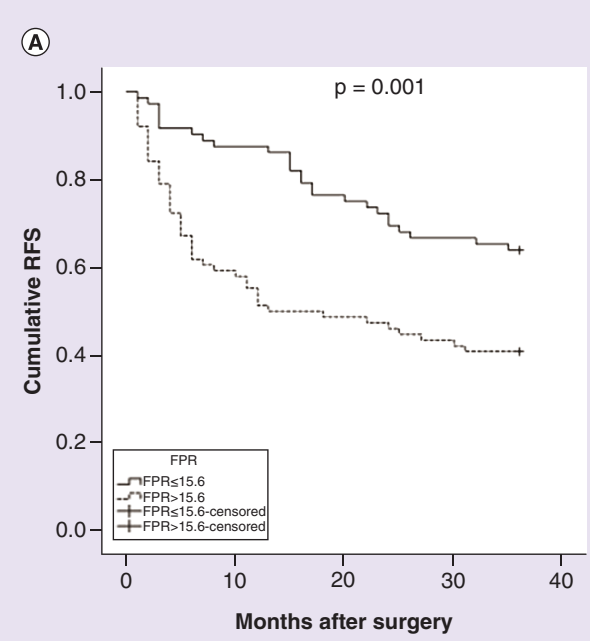

(B)
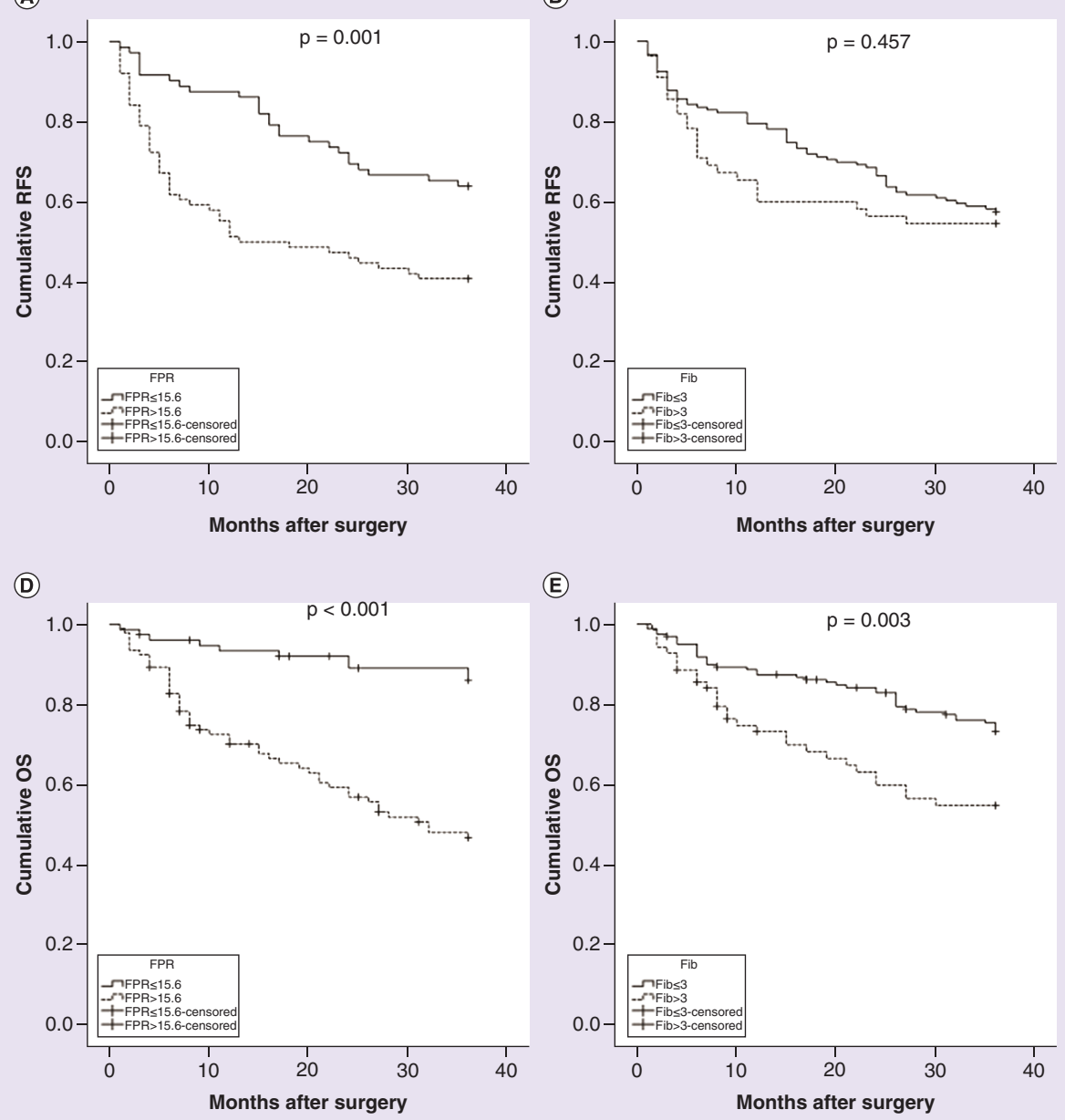

()

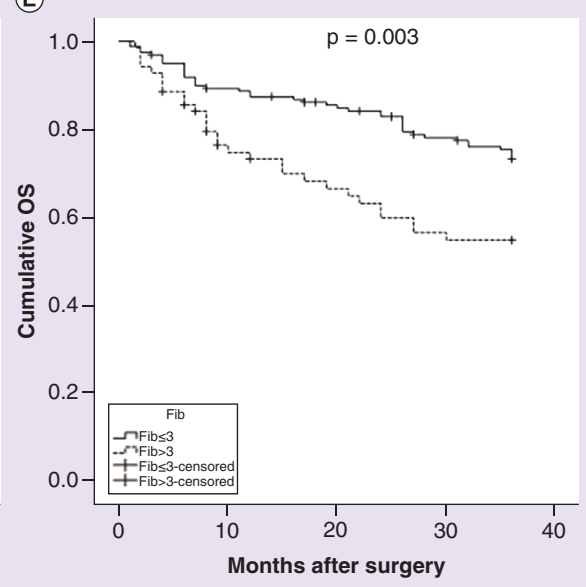

(c)

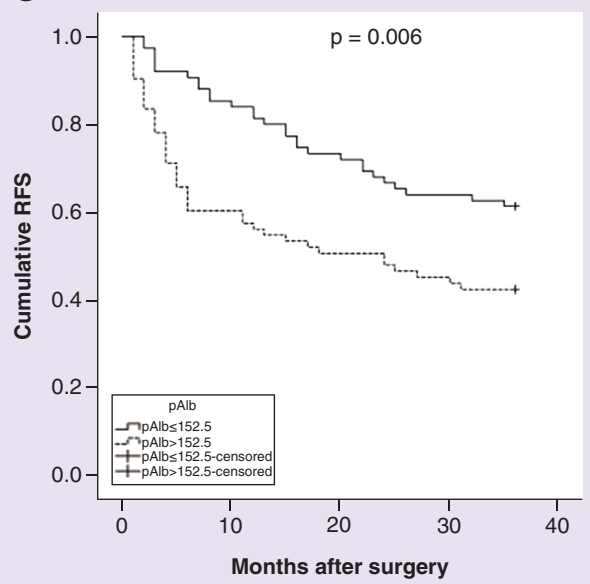

(F)

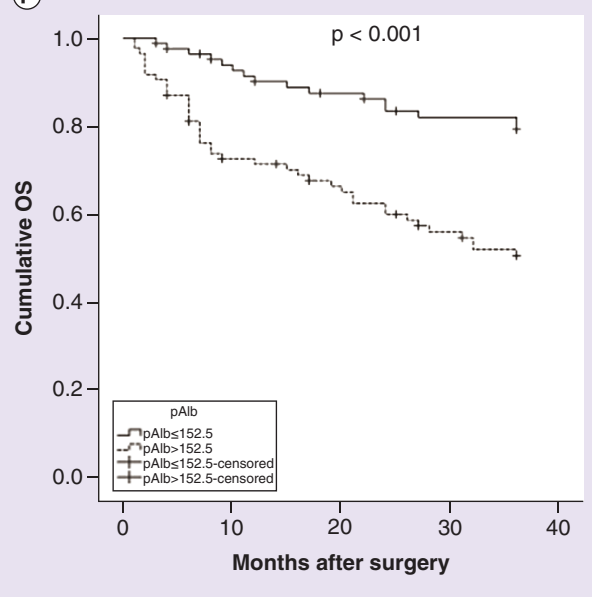

Figure 2. Kaplan-Meier curves of fibrinogen to prealbumin ratio for 3 years' overall survival and recurrence-free survival in hepatocellular carcinoma patients. (A) Kaplan-Meier curve of FPR for RFS; (B) Kaplan-Meier curve of Fib for RFS; (C) Kaplan-Meier curve of pAlb for RFS; (D) Kaplan-Meier curve of FPR for OS; (E) Kaplan-Meier curve of Fib for OS; (F) Kaplan-Meier curve of pAlb for OS. Fib: Fibrinogen; FPR: Fibrinogen to prealbumin ratio; OS: Overall survival; pAlb: Prealbumin; RFS: Recurrence-free survival.

\section{Discussion}

Predictive and prognostic biomarkers are key to evaluate clinical efficacy and outcome of HCC patient and to make decision to choose suitable therapeutic tool in clinic. Currently, BCLC stage and cell differentiation grade remain the most important and effective prognostic factors for the disease. However, the patients with the same pathological characteristics show significant heterogeneous prognosis. Therefore, it is necessary explore new and effective biomarkers to assess the progression of HCC.

In present study, we investigated the prognostic and predictive role of FPR in HCC patient and compared it with the other inflammatory biomarkers. We found that preoperative high FPR was significantly associated with large tumor size and advanced stage of HCC, suggesting that the biomarker could imply the aggressive phenotype of the disease. Moreover, circulating high FPR was obviously corrected with poor RFS and OS of surgical HCC patient, demonstrating that it was an independent prognostic factor for the cases. Additionally, the predicted efficacy of FPR was superior to the other inflammatory biomarkers, and the biomarker could identify the surgical patients who could benefit from adjuvant chemotherapy, suggesting that it was an effective predictive and prognostic biomarkers for surgical HCC patients.

Our previous study showed that preoperative circulating FPR could imply severity of chronic inflammation in solid malignancies [18], and the inflammatory reaction could influence the clinical efficacy of adjuvant chemother- 


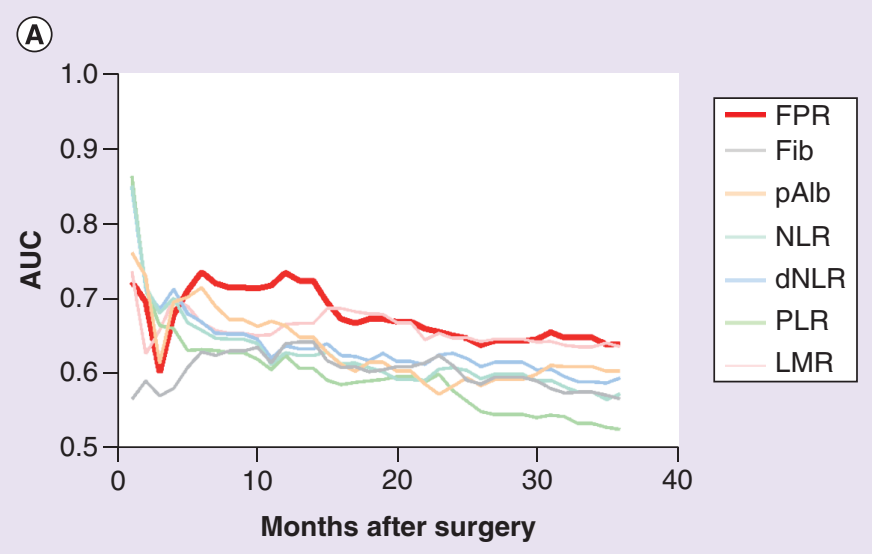

(B)

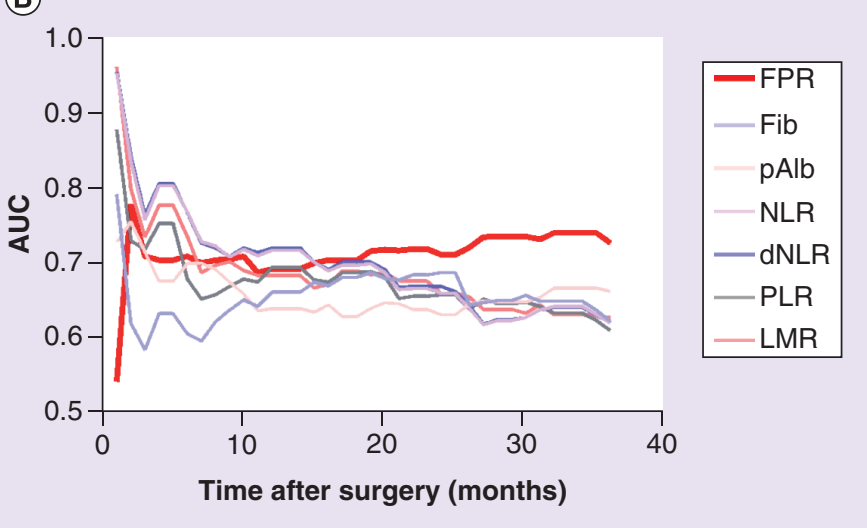

Figure 3. Time-dependent receiver-operating characteristic curves of preoperative circulating platelet to lymphocyte ratio, derived neutrophil to lymphocyte ratio, lymphocyte to monocyte ratio, neutrophil to lymphocyte ratio, fibrinogen, prealbumin and fibrinogen to prealbumin ratio for clinical outcome in hepatocellular carcinoma patients. (A) Time-dependent ROC curves for RFS; (B) time-dependent ROC curves for OS.

OS: Overall survival; RFS: Recurrence-free survival; ROC: Receiver operating characteristic.
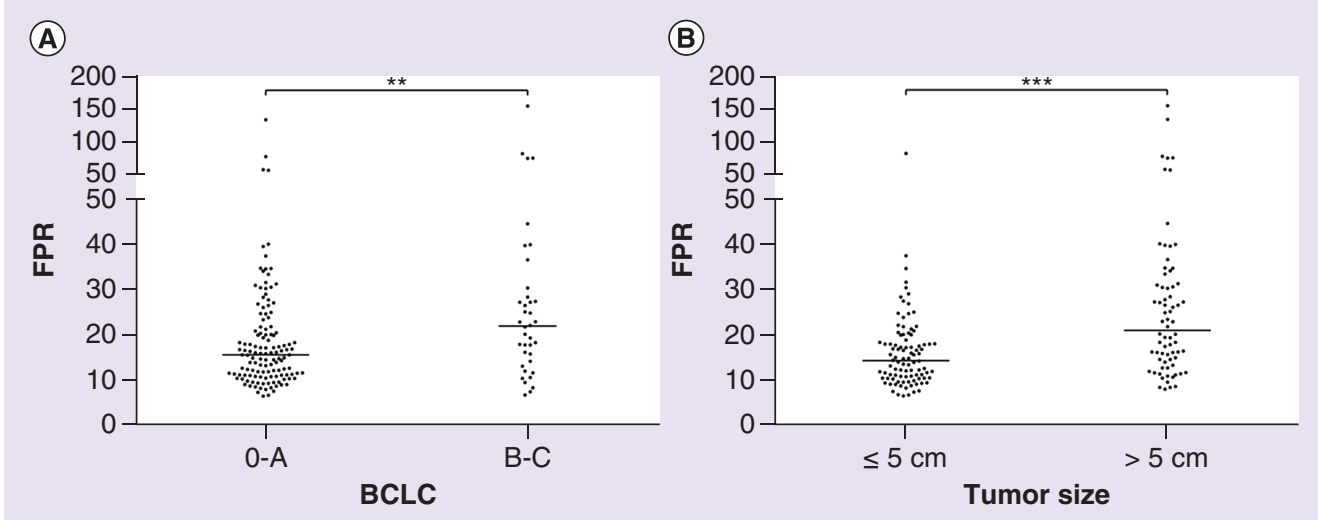

Figure 4. The correlation of fibrinogen to prealbumin ratio with Barcelona Clinic Liver Cancer group stage and tumor size in hepatocellular carcinoma patients. (A) FPR and BCLC stage; (B) FPR and tumor size.

$* * \mathrm{p}<0.01 ; * * * \mathrm{p}<0.001$.

BCLC: Barcelona Clinic Liver Cancer group; FPR: fibrinogen to prealbumin ratio.

apy [19]. This study showed the similar result and preoperative FPR could effective reflect the clinical efficacy of postoperative chemotherapy and prognosis of HCC patients. The following causes might explain our findings. First of all, cancer cell could synthesize and secret additional endogenous Fib [20], high Fib promoted the synthesis of IL-6 [21] and changed the nature of leucocyte infiltrate as well as stimulated T- and B-cells to promote chronic inflammatory response [22]. The bindings between Fib and members of the TGF-B, VEGF, PGF could both lead to angiogenesis, proliferation, metastasis of tumor cell and inhibited the apoptosis of the cancer cell [23]. Second, platelet-fibrin microthrombi acted as a physical barrier to prevent contact between natural killer cells and tumor cells to increase metastatic potential of tumor cell [24], and Fib acted as a bridging factor between tumor and host cell and enhanced the endothelial adhesion of tumor cell emboli in the vasculature of target organs [25]. Third, circulating pAlb could effectively reflect both chronic inflammation and nutrition in cancer patients, malnutrition was commonly observed in cancer patients [26] and nutritional status was significantly affected chemotherapy tolerance and survival of the patients [27].

This prospective study was investigate the predictive and prognostic role of circulating FPR in surgical HCC patients. It is an economic, simple, effective and promising biomarker to evaluate the survival of HCC patients and 

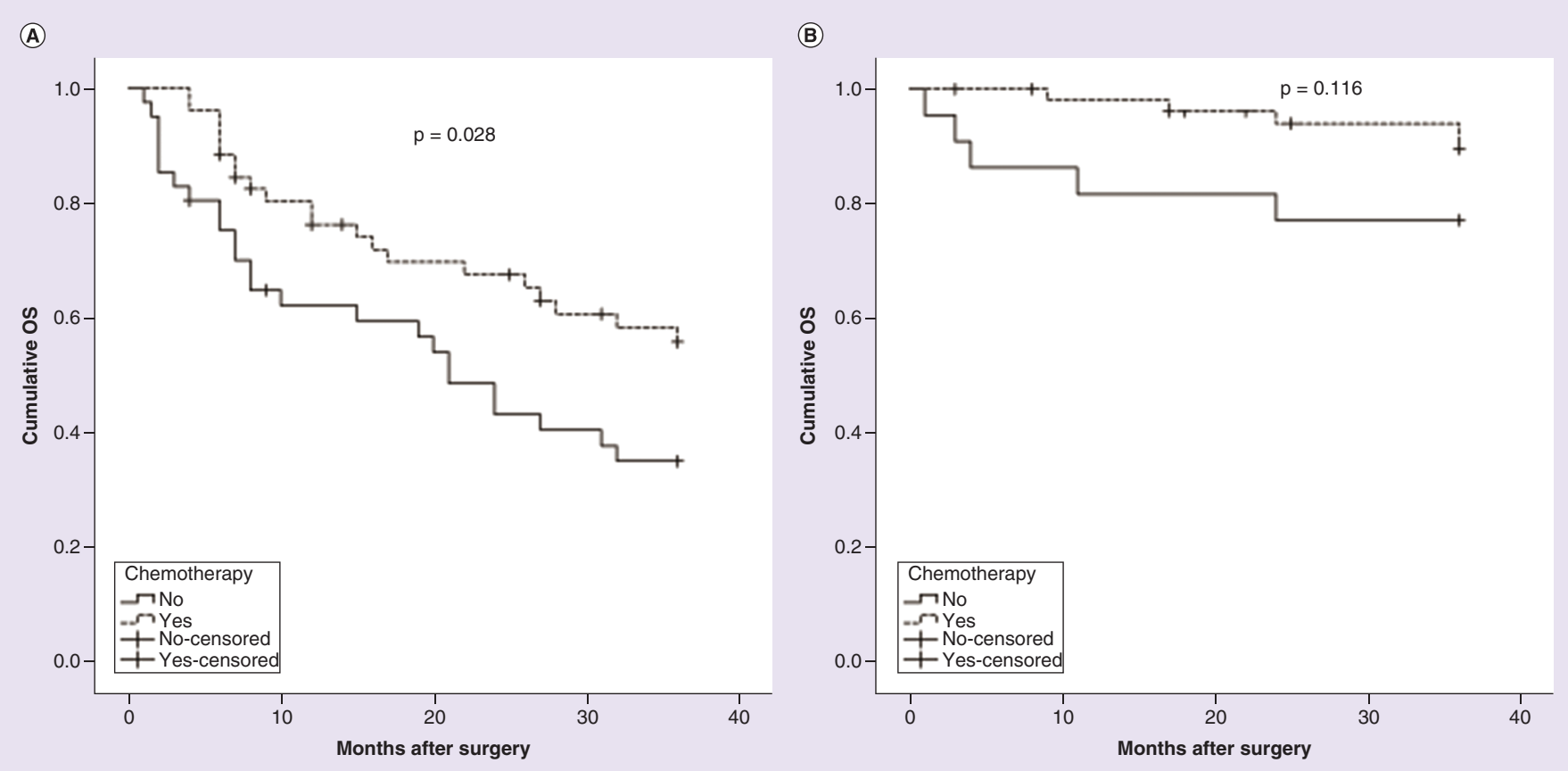

Figure 5. Kaplan-Meier curve of the patients with or without treatment of adjuvant chemotherapy after hepatectomy in high and low fibrinogen to prealbumin ratio subgroups. (A) Kaplan-Meier curve in high FPR group; (B) Kaplan-Meier curve in low FPR group. FPR: Fibrinogen to prealbumin ratio.

to distinguish the patients who can benefit from adjuvant chemotherapy. One the other hand, it is a single central design and small sample size study and only HCC patients who undergoing hepatectomy are enrolled. Therefore, the prognostic value of FPR needs to be validated in multicenter and large sample size prospective cohort study.

\section{Conclusion \& future perspective}

Our study indicated that preoperative FPR could serve as an independent prognostic biomarker for HCC patient and the patient with high FPR could benefit from adjuvant chemotherapy.

\section{Summary points}

- Surgical resection remains the main strategies for the treatment of hepatocellular carcinoma (HCC) patients. However, approximate $60-70 \%$ of patients were occurred recurrence in the following 5 years.

- Two hundred and thirty pathologically confirmed HCC individuals were enrolled in our study.

- Preoperative circulating neutrophils, lymphocytes, monocytes, platelet, fibrinogen (Fib) and prealbumin (pAlb) were measured.

- X-tile software, Kaplan-Meier curve, Cox regression, time-dependent receiver-operating characteristic (ROC) were used to evaluated prognostic roles of HCC.

- The Kaplan-Meier curve showed that Fib, pAlb, and Fib to pAlb ratio (FPR) were significantly associated with overall survival (OS) of HCC patients, while pAlb and FPR were significantly associated with recurrence-free survival (RFS) in HCC patients.

- Multivariate Cox regression showed that FPR was significantly associated with RFS and OS of HCC patients.

- The time-dependent ROC showed that area under curves of FPR reached up to 0.709 and 0.741 for predicting RFS and OS in 36th follow-up months, and it was higher than the area under curves of the other biomarkers in predicting recurrence and death within HCC patients.

- FPR could identify the surgical patients who could benefit from adjuvant chemotherapy.

- This is the first time to investigate the prognostic role of circulating FPR for HCC, while small sample size and simple center design were the limitation of our study. 
Financial \& competing interests disclosure

This study was supported by the Youth Fund of Education Department of Jiangxi Province (GJJ170155). The authors have no other relevant affiliations or financial involvement with any organization or entity with a financial interest in or financial conflict with the subject matter or materials discussed in the manuscript apart from those disclosed.

No writing assistance was utilized in the production of this manuscript.

\section{References}

Papers of special note have been highlighted as: $\bullet$ of interest; $\bullet \bullet$ of considerable interest

1. Chen W, Zheng R, Baade PD et al. Cancer statistics in China, 2015. CA Cancer J. Clin. 66(2), 115-132 (2016).

2. Cheng X, Sun P, Hu QG, Song ZF, Xiong J, Zheng QC. Transarterial (chemo)embolization for curative resection of hepatocellular carcinoma: a systematic review and meta-analyses. J. Cancer Res. Clin. Oncol. 140(7), 1159-1170 (2014).

3. Ao L, Song X, Li X et al. An individualized prognostic signature and multiomics distinction for early stage hepatocellular carcinoma patients with surgical resection. Oncotarget 7(17), 24097-24110 (2016).

4. Mantovani A, Allavena P, Sica A, Balkwill F. Cancer-related inflammation. Nature 454(7203), 436-444 (2008).

-• It shows the relationship between inflammation and cancer, which is the main theoretical basis of our research.

5. Colotta F, Allavena P, Sica A, Garlanda C, Mantovani A. Cancer-related inflammation, the seventh hallmark of cancer: links to genetic instability. Carcinogenesis 30(7), 1073-1081 (2009).

6. Balkwill F, Mantovani A. Inflammation and cancer: back to Virchow? Lancet 357(9255), 539-545 (2001).

7. Dolan RD, Lim J, McSorley ST, Horgan PG, McMillan DC. The role of the systemic inflammatory response in predicting outcomes in patients with operable cancer: systematic review and meta-analysis. Sci. Rep. 7(1), 16717 (2017).

8. Ji F, Liang Y, Fu SJ et al. A novel and accurate predictor of survival for patients with hepatocellular carcinoma after surgical resection: the neutrophil to lymphocyte ratio (NLR) combined with the aspartate aminotransferase/platelet count ratio index (APRI). BMC Cancer 16, 137 (2016).

- It illustrates the feasibility of inflammatory biomarkers as a prognostic factor for liver cancer.

9. Xu L, Yu S, Zhuang L et al. Systemic inflammation response index (SIRI) predicts prognosis in hepatocellular carcinoma patients. Oncotarget 8(21), 34954-34960 (2017).

10. Davalos D, Akassoglou K. Fibrinogen as a key regulator of inflammation in disease. Semin. Immunopathol. 34(1), 43-62 (2012).

- It shows the role of fibrin in inflammatory diseases.

11. Deng Q, He B, Liu X et al. Prognostic value of pre-operative inflammatory response biomarkers in gastric cancer patients and the construction of a predictive model. J. Transl. Med. 13, 66 (2015).

12. Steinbrecher KA, Horowitz NA, Blevins EA et al. Colitis-associated cancer is dependent on the interplay between the hemostatic and inflammatory systems and supported by integrin alpha(M)beta(2) engagement of fibrinogen. Cancer Res. 70(7), 2634-2643 (2010).

-• It reveals a unique link between fibrin (ogen) and the development of inflammation-driven malignancies.

13. Zhu LR, Li J, Chen P, Jiang Q, Tang XP. Clinical significance of plasma fibrinogen and D-dimer in predicting the chemotherapy efficacy and prognosis for small cell lung cancer patients. Clin. Transl. Oncol. 18(2), 178-188 (2016).

14. Gu W, Zhang G, Sun L et al. Nutritional screening is strongly associated with overall survival in patients treated with targeted agents for metastatic renal cell carcinoma. J. Cachexia Sarcopenia Muscle 6(3), 222-230 (2015).

15. Huang J, Wang Y, Yuan Y et al. Preoperative serum pre-albumin as an independent prognostic indicator in patients with localized upper tract urothelial carcinoma after radical nephroureterectomy. Oncotarget 8(22), 36772-36779 (2017).

16. Sanchez-Lara K, Turcott JG, Juarez E et al. Association of nutrition parameters including bioelectrical impedance and systemic inflammatory response with quality of life and prognosis in patients with advanced non-small-cell lung cancer: a prospective study. Nutr. Cancer 64(4), 526-534 (2012).

- It shows that nutritional status has an impact on the prognosis of the tumor.

17. Liaw YF, Kao JH, Piratvisuth T et al. Asian-Pacific consensus statement on the management of chronic hepatitis B: a 2012 update. Hepatol. Int. 6(3), 531-561 (2012).

18. Zhang J, Li SQ, Liao ZH et al. Prognostic value of a novel FPR biomarker in patients with surgical stage II and III gastric cancer. Oncotarget 8(43), 75195-75205 (2017).

19. Han Z, Jing Y, Xia Y et al. Mesenchymal stem cells contribute to the chemoresistance of hepatocellular carcinoma cells in inflammatory environment by inducing autophagy. Cell Biosci. 4, 22 (2014).

- It suggests that the microenvironment of inflammation may promote chemotherapy resistance.

20. Sahni A, Khorana AA, Baggs RB, Peng H, Francis CW. FGF-2 binding to fibrin(ogen) is required for augmented angiogenesis. Blood 107(1), 126-131 (2006). 
21. Ridker PM, Howard CP, Walter V et al. Effects of interleukin-1beta inhibition with canakinumab on hemoglobin A1c, lipids, C-reactive protein, interleukin-6, and fibrinogen: a Phase IIb randomized, placebo-controlled trial. Circulation 126(23), 2739-2748 (2012).

22. Gabay C. Interleukin-6 and chronic inflammation. Arthritis Res. Ther. 8(Suppl. 2), S3 (2006).

23. Martino MM, Briquez PS, Ranga A, Lutolf MP, Hubbell JA. Heparin-binding domain of fibrin(ogen) binds growth factors and promotes tissue repair when incorporated within a synthetic matrix. Proc. Natl Acad. Sci. USA 110(12), 4563-4568 (2013).

-• Fibrinogen causes angiogenesis, proliferation and metastasis of tumor cells and inhibited the apoptosis of the cancer cell.

24. Palumbo JS, Talmage KE, Massari JV et al. Platelets and fibrin(ogen) increase metastatic potential by impeding natural killer cell-mediated elimination of tumor cells. Blood 105(1), 178-185 (2005).

25. Yano HJ, Hatano K, Tsuno N et al. Clustered cancer cells show a distinct adhesion behavior from single cell form under physiological shear conditions. J. Exp. Clin. Cancer Res. 20(3), 407-412 (2001).

26. Jagoe RT, Goodship TH, Gibson GJ. Nutritional status of patients undergoing lung cancer operations. Ann. Thorac. Surg. 71(3), 929-935 (2001).

27. Shirai $Y$, Okugawa Y, Hishida A et al. Fish oil-enriched nutrition combined with systemic chemotherapy for gastrointestinal cancer patients with cancer cachexia. Sci. Rep. 7(1), 4826 (2017). 